\title{
Crecimiento económico en el sector agropecuario-silvícola a nivel nacional y regional en el período 1996-2008
}

\author{
The regional and national economic growth in the Chilean \\ agriculture-forestry sector, 1996-2008 \\ Ramón Valderas ${ }^{1}$, Mauricio Meyer $^{2}$, Rodrigo González ${ }^{3}$
}

\section{RESUMEN}

El sector agropecuario-silvícola ha demostrado un dinamismo positivo en las últimas décadas tanto en volumen, producción como en la diversificación de exportaciones y en el producto interno bruto (PIB). Existen, a pesar de los resultados globales nacionales, otras opiniones que estiman necesario darle atención a la desagregación del sector agropecuario-silvícola a niveles regionales y subsectoriales en su característica de producción primaria. Se plantea la afirmación de que la participación subsectorial y regional es decreciente en agricultura y que el crecimiento del sector aumenta su concentración y participación en algunas regiones del centro del país. En el estudio se concluye que actualmente los subsectores agricultura (cultivos), silvicultura, ganadería y fruticultura participan en un $16 \%, 18 \%, 24,8 \%$ y $40,7 \%$ respectivamente en el valor agregado del sector. Esto revela una disminución de la participación del subsector agricultura sobre la base de este parámetro. Respecto de las regiones, las que crecen a mayor velocidad en la participación son la Quinta, la Sexta, la Séptima y Región Metropolitana. Las regiones Primera, Segunda, Undécima y Duodécima son las que experimentan un estancamiento.

Palabras clave: Región, agricultura, ganadería, fruticultura, silvicultura, producto interno bruto.

\section{ABSTRACT}

The agricultural-forestry sector has shown a positive momentum in recent decades, both in volume production and in export diversification and gross internal product (GIP). In spite of the overall results, there are those who suggest that it is necessary to disaggregate the national economic agricultural-forestry sector into regional and sub-sector scales in terms of primary production. This includes the claim that in some regions crop agriculture is decreasing and the growth of the sector is concentrated in few regions of central Chile. The study concludes that currently the agricultural subsector (crops), forestry, livestock and fruit growing account for $15.9 \%, 18.5 \%, 24.8 \%$ and $40.7 \%$, respectively, of the gross internal product (GIP) of the sector. This reveals a declining share of the agriculture subsector on the basis of the GIP parameter. The Regions with faster increase in their GIP participation include the Fifth, Sixth, Seventh and Metropolitan Region. First, Second, Eleventh and Twelfth Regions remain without improvement. Key words: Region, agriculture, forestry, livestock, fruit growing.

\section{Introducción}

Existe discrepancia entre la Clasificación Internacional Industrial Uniforme (Naciones Unidas, 1990) y la posición de economistas que incluyen los encadenamientos, en especial hacia adelante, con otros sectores económicos ligados con lo agropecuario-silvícola. Este sesgo afecta la transparencia en cuanto a precisar la participación en el Producto Interno Bruto (PIB) del sector productivo ligado a la tierra. Este estudio pretende demostrar que a nivel nacional y regional es importante no perder de vista la actividad primaria, dados los numerosos actores que dependen de la agricultura en un sentido lato. Por un lado, está la gran cantidad de trabajadores y productores del sector y, por otro, está la reducida cantidad de empresas en la industria de alimentos y el reducido número de empleos que ocupa en relación al sector agropecuario-silvícola, lo que no puede ser ignorado desde un punto de vista analítico.

Por otra parte, en la segunda mitad del siglo XX se trabajó con mucho interés en concepciones que involucraban el desarrollo de la agricultura, la

\footnotetext{
Departamento de Economía Agraria, Facultad de Ciencias Agronómicas, U. de Chile, Santiago-Chile. E-mail: rvaldera@uchile.cl Departamento de Economía Agraria, Facultad de Ciencias Agronómicas, U. de Chile. Santiago-Chile. E-mail: mmeyer1@ vtr.net 3 Licenciado en Ciencias Agronómicas. Universidad de Chile. Santiago-Chile. E-mail: ignacio_mina@vtr.net
} 
ruralidad y el ordenamiento del territorio, considerando una equidad entre el crecimiento nacional y el crecimiento regional. Numerosos autores han realizado síntesis sobre esas materias, destacándose Sunkel (Sunkel O., 1970), Stohr (Stohr W., 1965), Williamson (Williamson G., 1965), Johnson (Johnson E. A., 1974), Siebert (Siebert H., 1969), Ben David (Ben David A., 1969, Kuklinski (Kuklinski A., 1972), y también Naciones Unidas (UN, 1974). En Chile, Boisier introdujo el modelo de Rotterdam, en una propuesta regional-nacional, considerando el período 1967-1971 para Chile (Boisier S., 1976). Posteriormente Valderas (Valderas R., 1988) describe modelos multirregionales, basados en minimización de costos de transporte o maximización de los valores agregados. En 1994, el Ministerio de Planificación Regional publica un trabajo denominado "Métodos y técnicas de análisis regional" donde se da cabida a nuevos procedimientos de toma de decisiones y análisis situacional, abandonándose la modelización con cálculos económicos estructurados. Valderas (Valderas R., 1997) remarca que a pesar de los avances en algunos campos, como los sistemas de información geográfica y el mejoramiento de las bases de datos estadísticos, el tratamiento de las prognosis y diagnósticos con orientación nacional-regional ha sido abandonado en el sector público. Se plantea, entonces, que "el país se encuentra ante el desafío de establecer subsistemas (regiones o macrorregiones) capaces de sobrevivir económica y físicamente, que en forma de combinación armónica puedan iniciar, consolidar y aumentar el crecimiento de cada uno y todos los subsistemas en el concierto nacional". Como es natural, el sector agropecuario-silvícola está vinculado a lo rural. En este mismo sentido, el Programa de Naciones Unidas para el Desarrollo (PNUD) en Chile ha elaborado algunos estudios centrados en lo que llaman el desarrollo humano, poniendo un cierto énfasis en las comunidades conectadas a un ámbito territorial (PNUD, 2005; PNUD, 2008).

Basado en las anteriores consideraciones se plantea un postulado principal: considerar el sector agropecuario-silvícola dentro de la economía regional y nacional como un marco para facilitar el entendimiento del desarrollo económico, en una óptica que busque un equilibrio equitativo de los componentes territoriales, rurales, económicos y nacionales.

Como una primera etapa en el logro del postulado anterior, se plantean como objetivos preliminares:
1) Estimar el aporte del sector y de los subsectores, en los períodos cubiertos por las compilaciones 1996 y 2003, expresado en su Producto Interno Bruto (PIB), en las líneas de tendencia y en la participación relativa entre los subsectores.

2) Estimar la participación regional dentro del PIB de cada región en los períodos cubiertos por las compilaciones 1996 y 2003, las líneas de tendencia y evolución de los PIB regionales, estableciendo las diferencias entre ellos respecto a su velocidad de crecimiento.

\section{Materiales y Métodos}

\section{Materiales}

Base de Datos SQL-Access de Censos Agropecuarios Nacionales.

Matriz Insumo-producto Compilación 1996 y 2003. Banco Central de Chile.

Estadísticas del Banco Central de Chile, Instituto Nacional de Estadísticas (INE).

Clasificación Internacional Industrial Uniforme (CIIU) Serie $\mathrm{M} \mathrm{N}^{\circ} 4$ Rev. (Comisión de Estadísticas de Naciones Unidas).

Software MQ Windows 2.

\section{Métodos}

Para el primer objetivo: Se estima la tendencia del PIB para el sector agropecuario-silvícola y para cada subsector a nivel país en los años disponibles para los períodos 1996-2003 (compilación Base 1996) y período 2003-2008 (Compilación Base 2003) respectivamente. Cada período se trata separadamente. Los resultados se expresan en porcentaje de variación anual del PIB y el PIB en miles de millones de pesos o en millones de pesos moneda constante. La estimación se realiza mediante mínimos cuadrados ordinarios, utilizando el software MQ Windows 2 (Render B. et al., 2006). Se utiliza la división de los subsectores que corresponde a la matriz insumo producto de 1996 para comparar los dos períodos analizados. Los subsectores son Agricultura (Cultivos), Fruticultura y el Resto. En la compilación 2003 se distinguen Agricultura, Fruticultura, Ganadería y Silvicultura.

Para el segundo objetivo: La división política administrativa utilizada considera 13 regiones, por lo tanto, la antigua primera región contiene a la actual primera región y la decimoquinta región; la 
décima región incluye a la actual décima región y la decimocuarta región, debido a que las informaciones económicas utilizadas en algunos casos no han separado las nuevas regiones de las antiguas.

Se estima la tendencia del PIB por cada región separadamente en los años disponible para los períodos 1996-2003 (compilación Base 1996) y período 2003-2008 (Compilación Base 2003) respectivamente. Los resultados se expresan en porcentaje de variación anual del PIB y el PIB en miles de millones de pesos o en millones de pesos moneda constante. La estimación se realiza mediante mínimos cuadrados ordinarios software MQ Windows 2 (Render B. et al., 2006). Se sigue la división política administrativa de Chile con trece regiones, sin considerar la nueva región XIV que está incluida en la X Región y sin considerar la nueva región XV que está incluida en la I Región para hacer comparables las bases estadística y de

Cuadro 1. Participación del sector: PIBs* en el PIB País y porcentaje de crecimiento anual (MM\$, 1996-2006).

\begin{tabular}{lllcc}
\hline Año & $\begin{array}{c}\text { PIB Sector } \\
(\mathrm{MM} \mathrm{\$ )**}\end{array}$ & $\begin{array}{c}\text { PIB País } \\
(\mathrm{MM} \mathrm{\$ )**}\end{array}$ & $\begin{array}{c}\text { Participación } \\
\text { Sector PIB } \\
(\%)\end{array}$ & $\begin{array}{c}\text { Crecimiento } \\
\text { Anual (\%) }\end{array}$ \\
\hline 1996 & 1323,5 & 29255,5 & 4,5 & - \\
1997 & 1345,5 & 31125 & 4,32 & 6,6 \\
1998 & 1412,5 & 32064,6 & 4,4 & 5,0 \\
1999 & 1401,5 & 31926 & 4,38 & $-0,8$ \\
2000 & 1485,9 & 32270 & 4,38 & 4,6 \\
2001 & 1576 & 34403 & 4,58 & 6,1 \\
2002 & 1647,6 & 35158 & 4,68 & 4,7 \\
2003 & 1748 & 36464,5 & 4,79 & 4,8 \\
\hline
\end{tabular}

* $\quad \mathrm{PIB}=$ PIB Sectorial

** MM\$, 1996

Fuente: De los autores con datos del Banco Central. Banco Central, 2010 construcción territorial de las matrices Insumo Producto.

\section{Resultados y Discusión}

\section{El sector agropecuario-silvícola a nivel nacional}

El comportamiento del sector revela una tendencia al crecimiento del PIB en el período 1996-2003, lo que se muestra en la Figura 1. El Cuadro 1 además presenta la información que permite concluir que para el mismo período el sector tiene una tasa de crecimiento anual promedio de un $5,3 \%$, exceptuando el año 1999, cuando hubo un valor negativo asociado a la crisis asiática ocurrida en ese entonces.

En el período 2003-2009 el sector agropecuariosilvícola a nivel nacional ha logrado mantener, con leve alza sostenida, su proporción en el PIB del país hasta el año 2009, aunque la tasa de crecimiento anual propia experimenta una caída de $0,68 \%$ anual en el período 2003-2009, véase el Cuadro 2. Por otra parte, experimenta una tasa de crecimiento positiva de $4,77 \%$ en el año 2009 , lo que se aprecia en la Figura 2.

\section{Los subsectores de lo agropecuario-silvícola}

La Figura 3 muestra la evolución en el período 1996-2003 de los subsectores. La fruticultura tiene un crecimiento sostenido, mientras para el Resto de los subsectores se presenta una tendencia al descenso. En el período 1996 a 2003 la agricultura (cultivos) no sigue una tendencia específica ya que el coeficiente de correlación entre tiempo y evolución del PIB es apenas de 0,25, rechazando una tendencia al alza; al recalcular la tendencia sobre la base de buscar

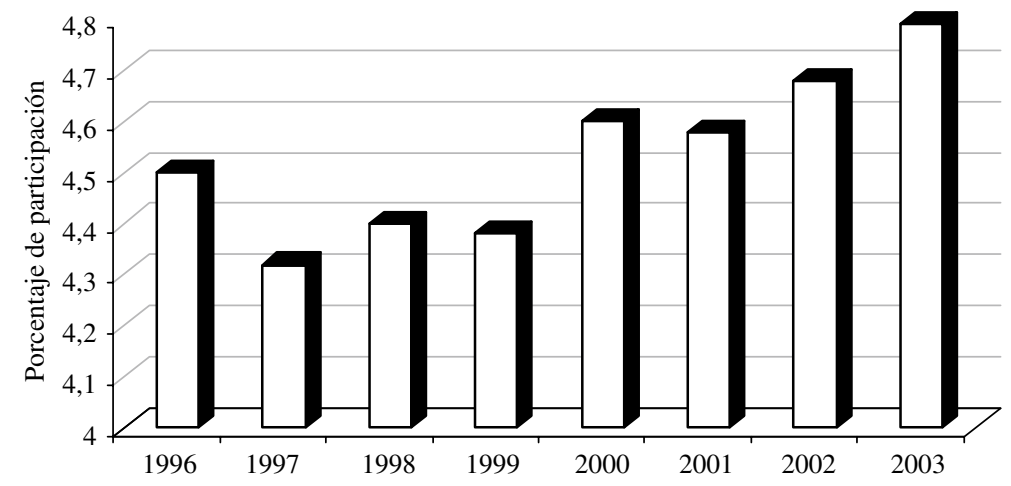

Figura 1. Evolución de la participación del PIB Agropecuario-Silvícola en el PIB Nacional (1996-2003). 
Cuadro 2. Participación sector: Porcentaje del PIBs* en el PIB y porcentaje Anual de Crecimiento (2003-2009).

\begin{tabular}{lcccc}
\hline Año & $\begin{array}{c}\text { PIB Sector } \\
(\text { MM\$)** }\end{array}$ & $\begin{array}{c}\text { PIB país } \\
(\text { MM\$)** }\end{array}$ & $\begin{array}{c}\text { Participación } \\
\text { Sector PIB } \\
(\%)\end{array}$ & $\begin{array}{c}\text { Crecimiento } \\
\text { Anual (\%) }\end{array}$ \\
\hline 2003 & 1842 & 51156 & 3,6 & - \\
2004 & 1994 & 52246 & 3,67 & 8,25 \\
2005 & 2179 & 57262 & 3,8 & 9,27 \\
2006 & 2323 & 59890 & 3,87 & 6,6 \\
2007 & 2339 & 62646 & 3,73 & 0,7 \\
2008 & 2409 & 64954 & 3,71 & 3 \\
2009 & 2524 & 63963 & $3,94 * *$ & 4,77 \\
\hline
\end{tabular}

* $\quad$ PIBs = Producto Interno Bruto Sectorial.

** Miles de millones de $\$ 2003$.

Fuente: De los autores con datos del Banco Central. Banco Central, 2010.

una línea paralela al eje del tiempo, es decir, una situación sin cambio, la línea de ajuste presenta un grado de significancia del 98\%. Complementando lo anterior, el Cuadro 3 presenta la ecuación de las líneas de tendencia con la pendiente y los coeficientes de correlación correspondientes.
La compilación actual 2003 divide el sector agropecuario-silvícola en cuatro subsectores: agricultura (cultivos), fruticultura, ganadería y silvicultura. Con el objeto de hacer una clasificación común, se considera en ambos casos la subdivisión el Resto, que agrupa ganadería y silvicultura. Al analizar los cuatro subsectores en el período 2003-2008 se advierte un descenso permanente del subsector agricultura, ver Figura 4; la agricultura presenta una pendiente negativa en la recta de tendencia de $-1,86 \%$ anual, pasando de $22,65 \%$ de participación sectorial en el 2003 a 15,9\% en el 2008. La participación de la ganadería y la silvicultura sumadas es superior a la fruticultura y su participación en el sector es creciente. La futicultura revela una evolución con crecimiento sostenido. Se muestra en la figura la línea de tendencia o pronóstico para la agricultura ajustada por el método de los mínimos cuadrados ordinarios. El Cuadro 4 muestra la evolución completa de los subsectores mediante la ecuación lineal con sus coeficientes de correlación, todos superiores al 77\%. También se incluyen los

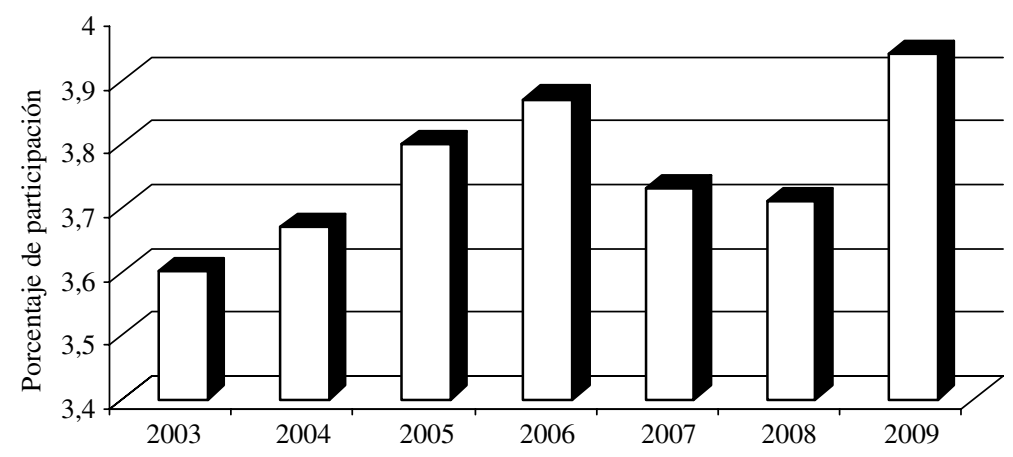

Figura 2. Evolución de la participación del PIB Agropecuario-Silvícola en el PIB nacional (2003-2009).

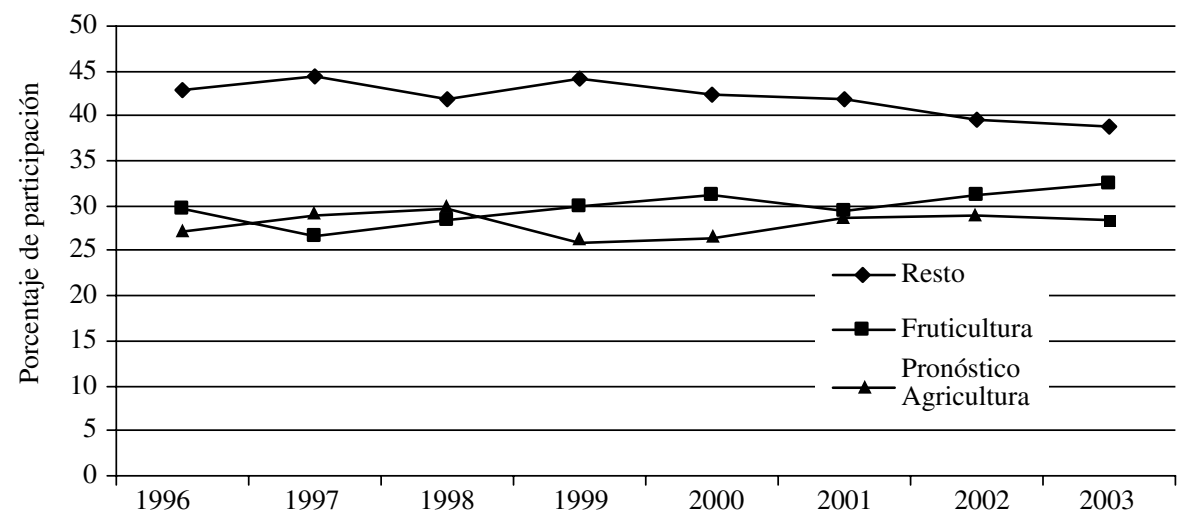

Figura 3. Evolución de la participación de los subsectores del sector Agropecuario-Silvícola en el Producto Interno Bruto del sector en porcentaje (1996-2003). 
Cuadro 3. Evolución en porcentajes a nivel del PIBs* de los subsectores Agricultura, Fruticultura y el resto (Periodo 1996-2003).

\begin{tabular}{clccccc}
\hline Subsector & Nombre & $\begin{array}{c}\text { Porcentaje } \\
1996\end{array}$ & $\begin{array}{c}\text { Porcentaje } \\
2003\end{array}$ & $\begin{array}{c}\text { Pendiente } \\
(\% \text { año })\end{array}$ & *Ecuación & $\begin{array}{c}\text { Coeficiente de } \\
\text { correlación }(\mathrm{r})\end{array}$ \\
\hline 1 & Agricultura & 27,2 & 28,5 & 0,11 & $\mathrm{Y}=27,49+0,11^{*}$ año & 0,25 \\
2 & Fruticultura & 29,7 & 31,9 & 0,53 & $\mathrm{Y}=27,43+0,53 *$ año & 0,78 \\
3 & Resto** & 43 & 39,6 & $-0,66$ & $\mathrm{Y}=44,99-0,65 *$ año & 0,86 \\
\hline
\end{tabular}

* $\quad$ PIBs $=$ Producto Interno Bruto Sectorial.

** Ganadería + Silvicultura.

*** Ajuste por mínimos cuadrados.

Fuente: Elaborado por los autores a partir de Cuentas Nacionales de Chile. Banco Central.

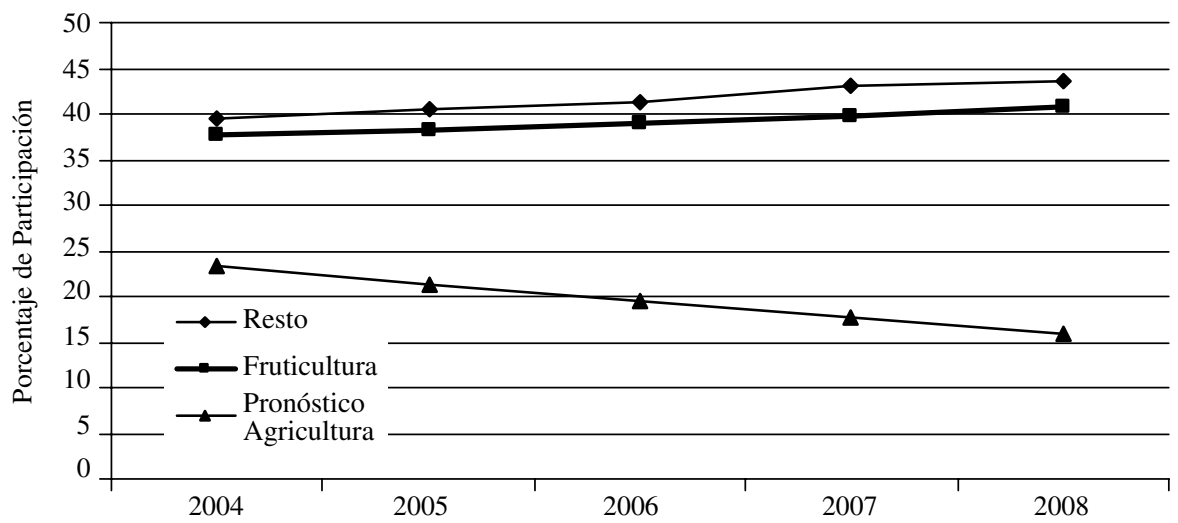

Figura 4. Evolución de la participación de los subsectores Agricultura, Fruticultura, Ganadería, Silvícola y resto en el PIB sectorial (2004-2008)

Cuadro 4. Evolución en porcentajes a nivel nacional del PIBs* de los subsectores Agricultura, Fruticultura, Ganadería y Silvicultura (Periodo 2003-2008).

\begin{tabular}{|c|c|c|c|c|c|c|}
\hline Subsector & Nombre & $\begin{array}{c}\text { Porcentaje } \\
1996\end{array}$ & $\begin{array}{c}\text { Porcentaje } \\
2003\end{array}$ & $\begin{array}{l}\text { Pendiente } \\
\text { (\% anual) }\end{array}$ & Ecuación** & $\begin{array}{l}\text { Coeficiente de } \\
\text { correlación (r) }\end{array}$ \\
\hline 1 & Agricultura & 22,65 & 15,9 & $-1,86$ & $\mathrm{Y}=25,1-1,86 *$ año & 0,98 \\
\hline 2 & Fruticultura & 37,75 & 40,8 & 0,77 & $\mathrm{Y}=36,78+0,77 *$ año & 0,98 \\
\hline 3 & Ganadería & 23,5 & 24,8 & 0,41 & $\mathrm{Y}=22,49+0,41 *$ año & 0,77 \\
\hline 4 & Silvicultura & 16,1 & 18,5 & 0,63 & $\mathrm{Y}=15,85+0,63 *$ año & 0,87 \\
\hline 5 & Resto $(3+4)$ & 39,6 & 43,6 & 1,07 & $\mathrm{Y}=38,36+1,07 *$ año & 0,98 \\
\hline
\end{tabular}

* $\quad$ PIBs = Producto Interno Bruto Sectorial

** Ajuste por mínimos cuadrados.

Fuente: Elaborado por los autores a partir de Cuentas Nacionales de Chile. Banco Central.

porcentajes de participación de cada subsector para 2003 y 2008 dentro del sector.

\section{El sector agropecuario-silvícola en las regiones}

La Figura 5 y Figura 6 permiten observar la diferencia de magnitudes del PIB de regiones de distintas latitudes, expresada respectivamente en millones de pesos de 1996 y 2003. Se confirma el alto valor del PIB en las regiones centrales del país (V, Región Metropolitana, Sexta y Séptima), manteniendo una alta tasa de crecimiento comparativa; la participación de este conjunto alcanza al 62,08\% del PIB del 2008. El otro gran aporte al sector se agrupa entre la VIII, IX y X regiones con un 30,55\% en el PIB de 2008, lo que replica proporciones parecidas entre 1996 


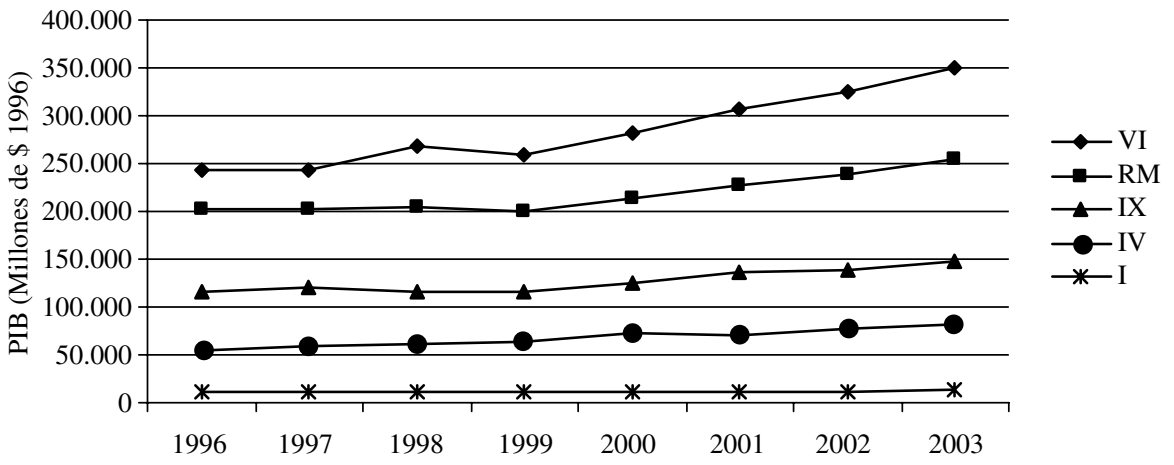

Figura 5. Crecimiento del Sector Agropecuario-Silvícola por Regiones.

Período 1996-2003 (Millones de \$ de 1996).

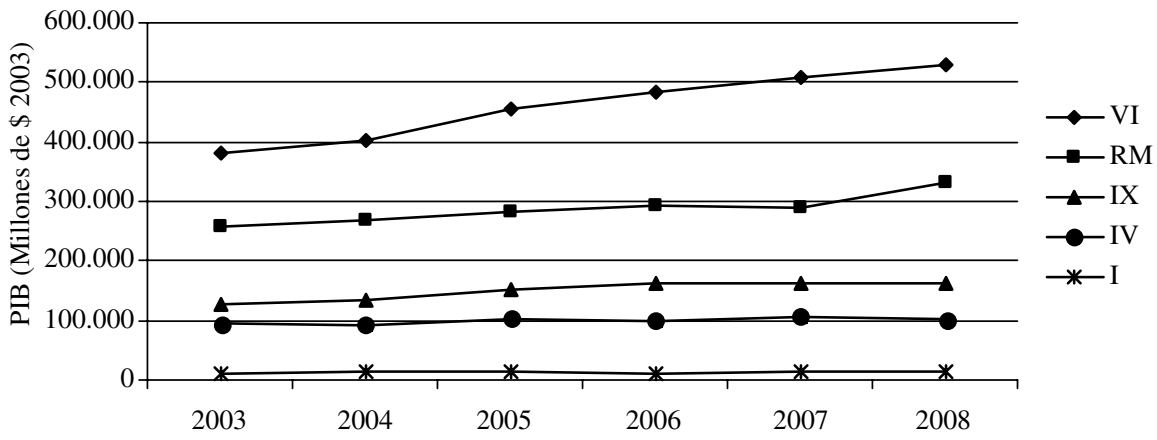

Figura 6. Crecimiento del Sector Agropecuario-Silvícola por Regiones.

Período 2003-2008 (Millones de \$ de 1996).

Cuadro 5. Crecimiento del Sector Agropecuario-Silvícola por Regiones. período 1996-2003. (Millones de pesos de 1996).

\begin{tabular}{crrclc}
\hline Región & PIB 1996 & PIB 2003 & $\begin{array}{c}\text { Pendiente } \\
\text { (MM/anuales) }\end{array}$ & *Ecuación & $\begin{array}{c}\text { Coeficiente de } \\
\text { correlación }(\mathrm{r})\end{array}$ \\
\hline I** & 11.185 & 12.854 & 182,7 & $\mathrm{Y}=11253+182 *$ año & 0,92 \\
II & 1.474 & 1.797 & 40,45 & $\mathrm{Y}=1441+40 *$ año & 0,98 \\
III & 26.396 & 31.021 & 570,38 & $\mathrm{Y}=25487,5+570 *$ año & 0,85 \\
IV & 55.000 & 82,427 & 3,83 & $\mathrm{Y}=505708+3,83 *$ año & 0,98 \\
V & 113.666 & 197.740 & 12845 & $\mathrm{Y}=92323+12845 *$ año & 0,98 \\
VI & 243.279 & 351.070 & 1566 & $\mathrm{Y}=214119+1566 *$ año & 0,96 \\
VII & 173.946 & 233.661 & 8122 & $\mathrm{Y}=164809+8122 *$ año & 0,91 \\
VIII & 198.846 & 232.214 & 4271 & $\mathrm{Y}=189878+4271 *$ año & 0,88 \\
IX & 115.953 & 147.853 & 4602 & $\mathrm{Y}=106175+4602 *$ año & 0,89 \\
X** & 154.151 & 185.261 & 3361 & $\mathrm{Y}=159791+3361 *$ año & 0,84 \\
XI & 8.325 & 8.229 & 27,46 & $\mathrm{Y}=8594-27 *$ año & 0,37 \\
XII & 7.960 & 7.711 & 45,1 & $\mathrm{Y}=8146-45 *$ año & $-0,55$ \\
RM & 203.371 & 225.244 & 5042 & $\mathrm{Y}=191781+5042 *$ año & 0,85 \\
\hline
\end{tabular}

* Ajuste por mínimos cuadrados.

** Según antigua división administrativa. 
Cuadro 6. Crecimiento del Sector Agropecuario-Silvícola por Regiones. Periodo 2003-2008.

(Millones de pesos de 2003).

\begin{tabular}{lrrccc}
\hline Región & $\begin{array}{r}\text { PIBs* } \\
2003\end{array}$ & $\begin{array}{c}\text { PIBs* } \\
2008\end{array}$ & $\begin{array}{c}\text { Pendiente } \\
(\% \text { anual })\end{array}$ & Ecuación*** & $\begin{array}{c}\text { Coeficiente de } \\
\text { correlación (r) }\end{array}$ \\
\hline I** & 11295 & 12585 & 125 & $\mathrm{Y}=11861+125 *$ año & 0,38 \\
II & 1671 & 1696 & 37 & $\mathrm{Y}=1538+37 *$ año & 0,51 \\
III & 37037 & 47398 & 2414 & $\mathrm{Y}=33433+2414 *$ año & 0,97 \\
IV & 94502 & 104015 & 2238 & $\mathrm{Y}=91506+2238 *$ año & 0,76 \\
V & 195422 & 282751 & 18040 & $\mathrm{Y}=172694+18040 *$ año & 0,99 \\
VI & 263481 & 349394 & 31472 & $\mathrm{Y}=350598+31472 *$ año & 0,98 \\
VII & 263481 & 349394 & 17331 & $\mathrm{Y}=269575+17331 *$ año & 0,82 \\
VIII & 279315 & 333932 & 12707 & $\mathrm{Y}=280362+12707 *$ año & 0,78 \\
IX & 126146 & 162096 & 7674 & $\mathrm{Y}=123180+7674 *$ año & 0,92 \\
X** & 185182 & 240615 & 9372 & $\mathrm{Y}=193793+9372 *$ año & 0,82 \\
XI & 6722 & 7884 & 272 & $\mathrm{Y}=6560+272 *$ año & 0,89 \\
XII & 3077 & 3822 & 155 & $\mathrm{Y}=3011+155 *$ año & 0,88 \\
RM & 258607 & 332500 & 12892 & $\mathrm{Y}=241979+12892 *$ año & 0,87 \\
\hline
\end{tabular}

* $\quad$ PIBs = Producto Interno Bruto Sectorial.

** Según antigua división administrativa.

*** Ajuste por mínimos cuadrados.

Fuente: Elaborado por los autores a partir de Cuentas Nacionales de Chile. Banco Central.

Cuadro 7: Agrupación de las regiones según participación y tendencia de crecimiento del PIBs*.

\begin{tabular}{lcccc}
\hline \multicolumn{1}{c}{ Región } & $\begin{array}{c}\text { Participación en el PIB } \\
(\% / \text { año 2009) }\end{array}$ & $\begin{array}{c}\text { Crecimiento PIB\% anual. } \\
1996-2003\end{array}$ & $\begin{array}{c}\text { Crecimiento PIB\% anual. } \\
2003-2008\end{array}$ & $\begin{array}{c}\text { Tipo de } \\
\text { Crecimiento }\end{array}$ \\
\hline I + XV & 0,52 & $+2,12$ & $+2,28$ & Bajo \\
II & 0,01 & $+2,98$ & $+0,3$ & Bajo \\
III & 1,94 & $+2,82$ & $+5,58$ & Regular \\
IV & 4,30 & $+7,14$ & $+2,01$ & Regular \\
V & 11,76 & $+10,56$ & $+8,93$ & Alto \\
R.M. & 13,8 & $+1,53$ & $+5,71$ & Alto \\
VI & 22,1 & $+6,32$ & $+7,95$ & Alto \\
VII & 14,5 & $+4,9$ & $+6,52$ & Alto \\
VIII & 13,85 & $+2,43$ & $+3,9$ & Regular \\
IX & 6,72 & $+3,93$ & $+6,0$ & Regular \\
X + XIV & 9,98 & $+2,88$ & $+5,98$ & Regular \\
XI & 0,37 & $-0,16$ & $+3,45$ & Bajo \\
XII & 0,16 & $-0,44$ & $+4,8$ & Bajo \\
\hline
\end{tabular}

* $\quad$ PIBs $=$ Producto Interno Bruto Sectorial.

Fuente: Elaborado por los autores a partir de Cuentas Nacionales de Chile. Banco Central.

y 2008. La información sintetizada para cada una de las regiones se encuentra en los Cuadros 5 y 6 , donde se consignan los cambios de la composición del sector por regiones, complementados con las tendencias y los coeficientes de correlación entre el PIB y el tiempo.

Del porcentaje restante, la mayor parte la comparten la III y IV regiones con un $6,28 \%$ del total. Las regiones extremas acumulan un $1,09 \%$ del sector al año 2008.
Estos resultados en cuanto al porcentaje de participación de cada región en el PIB sectorial permiten plantear una división de las regiones en macrozonas según la intensidad del crecimiento económico y la participación relativa. El Cuadro 7 revela, según los indicadores usados en el cuadro, las siguientes divisiones como macrozonas: Crecimiento bajo, la zona del Norte Grande: I, II y XV; crecimiento regular, la zona del Norte Chico: III y IV regiones; crecimiento alto, la Zona Centro con la V, Región Metropolitana, VI y VII 
regiones; crecimiento regular, la zona Centro Sur con la VIII, IX, X y XIV regiones; y crecimiento bajo para la zona del Extremo Sur con la Undécima y Duodécima regiones.

Los períodos 1996-2003 y 2003-2008 se han presentado separados, ya que la información elaborada por las Cuentas Nacionales de Chile están limitadas en su validez actualmente a lapsos menores de diez años, deseablemente quinquenales. La argumentación principal para separar los períodos es que el sistema de cuentas nacionales se funda en que los coeficientes técnicos utilizados por la matriz Insumo-producto son constantes y además supone que la relación productos intermedios y productos finales es de orden lineal. Estas limitaciones, originadas por la aplicación del Modelo de Leontief (Dorfman et al., 1964), obligan a reconocer que los cambios estructurales en tecnología, demandas, inversiones y otros llevan a la necesidad de recalcular las matrices y aplicar entonces un cambio en las compilaciones tratando de normalizarlas cada cinco años. De esta manera la base 1996 duró ocho años y se espera un cambio inminente de sustitución de la base 2003. Además, en el caso del sector agropecuario-silvícola se han traspasado servicios agrícolas o productos industriales a otros sectores, dentro de la clasificación CIIU (por ejemplo, Maquinaria agrícola, o algunas agroindustrias no prediales), lo que hace que por esta causa se reduzca la participación del sector. Además, entre la compilación 1996 y 2003 hubo que poner en práctica las recomendaciones de los expertos sobre el sector, lo que hace que ambas bases sean diferentes (Seruzier M., 2001).

Este caso de estudio posee dos series que tienen 8 puntos y 6 puntos en el tiempo respectivamente. Una serie de tiempo normalmente necesita mayor número de valores para hacer un estudio estadístico-econométrico suficientemente respaldado. En nuestra situación no se cumple con esa condición, la que no es relevante al tratarse en el fondo de estudiar solamente una sola población de actores económicos o un solo universo con una medida común: el Producto Interno Bruto y/o su valor agregado como sector. Los procedimientos de las Cuentas Nacionales apuntan a medir exhaustivamente la economía nacional tratándola como una identidad, por lo tanto, su fortaleza está en eso mismo. Mientras mida adecuadamente este cuerpo económico chileno, la información resultante no merece críticas de fondo. Sin embargo, el tema se traslada a la medición. Esta medición está respaldada internacionalmente por la clasificación de las actividades (CIIU) y por los métodos de obtención y tratamiento de las conciliaciones y a que se puede acceder razonablemente a la base nacional de informantes. Algunos saltos en las mediciones resultantes del componente agrícola resultan de conocer mejor el monto de transacciones reales que no quedan registradas monetariamente y por otro lado lleva a eliminar actividades que no pertenecen al sector no obstante que lo agropecuario-silvícola muchas veces es prácticamente indivisible con los servicios o con la agroindustria conectados a ella. No hay duda de que las series cortas pueden medir las crisis por un lado y garantizar la validez de los coeficientes técnicos. A la luz de los resultados que aparecen en el Cuadro 1 se verifica que el sector agropecuario-silvícola no sólo ha logrado mantener su participación en el PIB nacional sino que llegó a incrementarlo en el 2009.

Respecto, al efecto amortiguamiento que se atribuye al sector frente a choques externos (O'Ryan R. y Miller S., 2005) los resultados obtenidos coinciden con una resistencia a bruscos cambios en la economía. Esto tiene una conexión con lo que comienza a llamarse una agricultura resiliente. En efecto, desde 2008 la economía chilena ha estado sometida a los efectos de una crisis global que afecta los mercados juntos, también a los cambios del precio del dólar y del petróleo. En los últimos años ha pasado el sector agropecuario-silvícola desde un $3,6 \%$ de participación a 3,9\% en el PIB del país (ver Cuadro 1), detentando un crecimiento del PIB en $4,7 \%$ en el 2009 que comparado con sectores como Minería que baja en 1,4\%, la Industria un $7 \%$, la Construcción un $5,2 \%$ y el país en su conjunto, un $1,5 \%$ en ese mismo año testimonia una capacidad de reaccionar y esto especialmente considerando los resultados de nuestro intercambio comercial con el exterior (Valderas, et al., 2006; Valderas, et al., 2010). Este resultado no oculta, sin embargo, que lo agropecuario-silvícola también se ha resentido por la crisis externa, pero sin dejar de crecer. El Cuadro 2 ofrece una tasa normal de crecimiento del sector exceptuando el año 1999 con una contracción del aporte sectorial. Los antecedentes económicos del país y del mundo en ese año revelan la primera crisis global, llamada la crisis asiática, que afectó precios y cumplimiento de pagos a la agricultura y a la silvicultura.

Otro caso es la situación preocupante de las regiones extremas del país, que presentan un PIB 


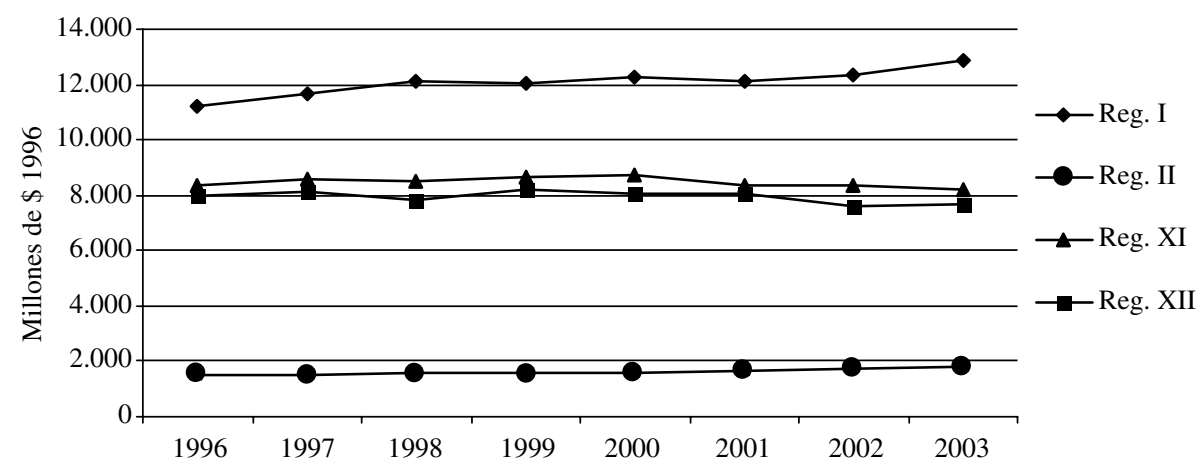

Figura 7. Crecimiento del Sector Agropecuario-Silvícola en regiones I, II, XI y XII. (1996-2003) Millones de \$ 1996.

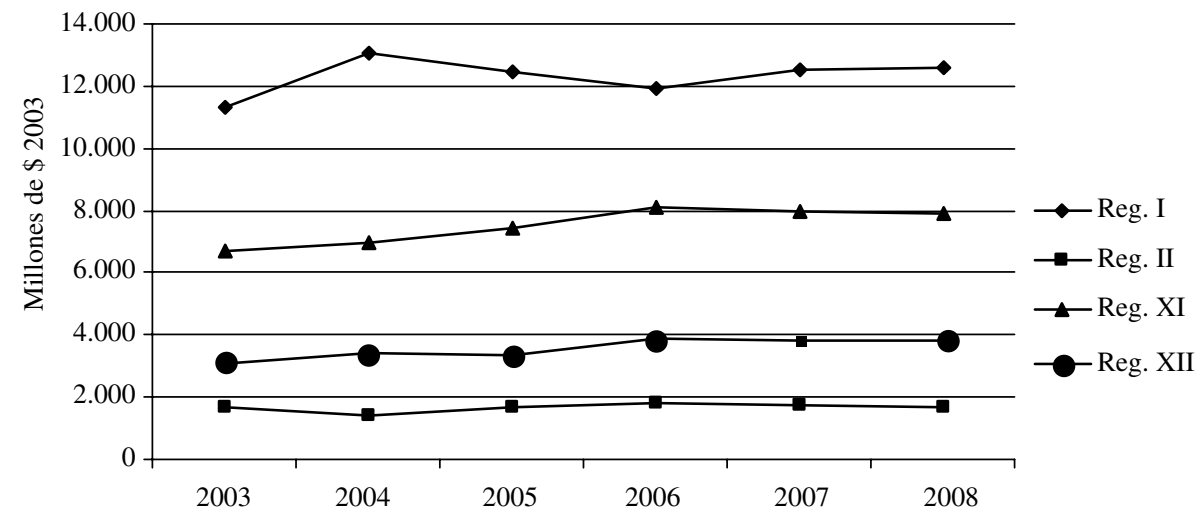

Figura 8. Crecimiento del Sector Agropecuario-Silvícola en regiones I, II, XI y XII. (2003-2008) Millones de \$ 2003.

sectorial per cápita muy inferior al resto de las regiones del país. Las Figuras 7 y 8 presentan una situación no sólo, lo que es conocido, de pequeña participación en el valor agregado del sector, sino que se puede observar que su comportamiento histórico se puede catalogar como estancamiento. La Figura 9 corrobora la situación respecto al nivel de aporte de la agricultura en PIB por persona en las regiones extremas, expresado en el Producto Interno Bruto del sector en las respectivas regiones per cápita en moneda de 2003. En efecto, las regiones extremas del país detentan un valor agregado per cápita anual inferior a 0,6 millones de pesos de 2003 en el 2008, en cambio, las otras regiones perciben un valor agregado per cápita anual que va desde 1 millón de pesos hasta sobre 2 millones de pesos (moneda 2003) per cápita al año 2008. Aunque pudieran aducirse consideraciones climáticas, edáficas y topográficas para justificar esta situación, no es menos cierto que habría que buscar también consideraciones de orden de conservación de la naturaleza, de la obligación actual de darle prioridad a las minorías étnicas (ya sea como una reparación del pasado (Duodécima Región) o por la situación actual regiones I, II y $\mathrm{XV}$ ), razones geopolíticas y razones económicas de aprovechamiento de los factores agua, suelo. Todo esto sin entrar en el horizonte de largo plazo que hace imperativo sopesar el papel futuro de estas áreas limítrofes con acceso al mar, con un papel importante hacia el Asia Pacífico; sopesar también las extensas superficies disponibles para actividades que tiendan a devolver la fertilidad en esas superficies, teniendo presente los avances tecnológicos que pueden asegurar la sostenibilidad de una actividad económica, comercial en climas que aunque difíciles son más benignos que en otras latitudes iguales del hemisferio Norte donde han encontrado soluciones y posibilidad de expandir su calidad de vida. La nación tiene una deuda con los mestizos, descendientes de pueblos diezmados, y con la memoria de los que se extinguieron en las regiones australes (Peri R., 1975). Por otro, lado en la Región Arica y Parinacota (Región XV) el caso de la cuenca del río Lluta puede ser emblemático en el sentido de que subsisten allí 


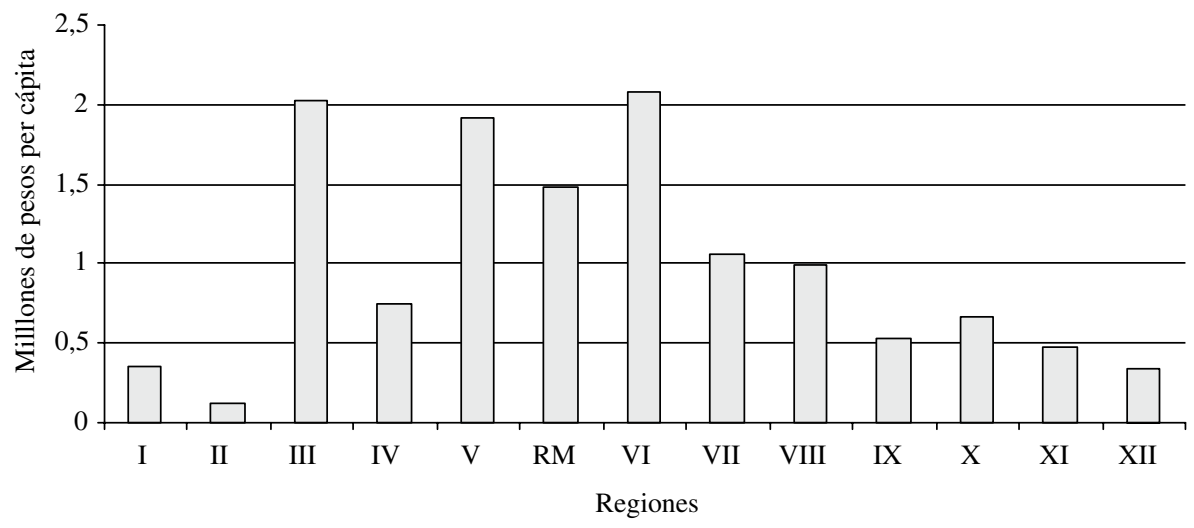

Figura 9: Producto Interno Bruto por Habitante Rural en Chile. (Millones de \$ 2003 per cápita).

variados problemas como el manejo del humedal en la desembocadura desde el punto de vista del puerto y de la conservación de especies; la minería genera preocupaciones que aún tienen repercusiones internacionales por la fragilidad del sistema hídrico y sus limitaciones actuales (Torres A. y Acevedo E., 2005; Visión Universitaria, 2009; diarios de Internet y Junta de vigilancia del valle del Lluta, entre otros); y la presencia de la población aimara en el sector rural especialmente su ingreso económico genera una preocupación de equidad.

\section{Conclusiones}

\section{Respecto del primer objetivo}

El sector agropecuario-silvícola conserva una capacidad de absorción de impactos externos en el corto plazo superior a otras actividades tradicionales.

El subsector agricultura (cultivos) ha ido perdiendo terreno frente a lo otros subsectores, los cuales lo superan en participación dentro del sector que en orden creciente participan a 2008 la Silvicultura con un $18,5 \%$, Ganadería con un $24,8 \%$ y Fruticultura con $40,8 \%$ del producto agropecuario silvícola.

\section{Respecto del segundo objetivo}

\section{En lo regional}

La I, XV, II, XI y XII regiones han tenido un desarrollo inferior al resto de las otras regiones en el lapso 1996-2008.

Las regiones III y IV han tenido períodos dinámicos y relativamente satisfactorios en períodos cruzados, ubicándose entre las regiones dinámicas y las de menor desarrollo económico.

Las regiones V, R.M., VI, VII han tenido crecimientos que a nivel nacional se pueden clasificar como dinámicos.

Las regiones VIII, IX, XIV y X han obtenido avances inferiores a las regiones dinámicas, pero a un nivel satisfactorio en el lapso 1996-2008.

La regiones XI y XII tienen un nivel de crecimiento promedio insatisfactorios sin embargo, se detecta recuperación en período 2003-2008 que apenas compensa el retroceso de 1996-2003.

El grupo de mayor dinamismo se concentra en la zona central con un $62 \%$, seguido de la Zona Centro sur con un 30,55\% del PIB agropecuariosilvícola. 


\section{Literatura Citada}

Banco Central

2006 Cuentas Nacionales de Chile 1996-2005. Mayo 2006.

Santiago. Chile.

2006 Cuentas Nacionales de Chile. Compilación de referencia 2003.

2009 Cuentas Nacionales de Chile 2003-2009. Mayo 2009.

Santiago. Chile.

Ben David, A.

1972 The concept-Strategy-Projects Approach to planning for regional development, International Development Review 1972/1.

Boisier, $\mathrm{S}$.

1976 Diseño de planes regionales, Colegio oficial de ingenieros de Caminos, Canales y Puertos, Centro de perfeccionamiento, Madrid, España. p. 299.

Dorfman, R.; Samuelson P. y Solow, R.

1964 Programación lineal y análisis económico, Aguilar S.A. de Ediciones, Madrid.

Instituto Nacional de Estadísticas. Chile (INE)

1997 VI Censo Agropecuario.

2007 VII Censo Agropecuario.

Johnson, E.A.J.

1974 The organization of space in developing countries, Harvard University Press, Cambridge.

Kuklinski, A.

1972 Growth poles and growth centres in regional planning. The Hague. Mouton.

Naciones Unidas 1990 Clasificador Industrial Uniforme (CIIU Rev.3). Comisión Estadística NU. Serie M, Re.3.

Naciones Unidas

1974 Planificación Regional. Conclusiones y recomendaciones del proyecto DP/UN/BOL/ 71010/4 Nueva York.

O’Ryan, R. y Millar, S.

2005 "El rol de la agricultura en la mitigación de la pobreza, la distribución del ingreso y el desarrollo económico". En Valdés A. y Foster W., 2005, Externalidades de la Agricultura chilena, Ediciones U. Católica Chile. p. 290.

Peri, R.

1975 Los genocidas, en "Concurso Literario", ODEPLAN, Chile, pp. 126-214.

Programa de las Naciones Unidas para el Desarrollo (PNUD) 2005 Serie Temas de Desarrollo Humano sustentable, $N^{\circ} 12$ Santiago, Chile.
PNUD

2008 Desarrollo Humano en Chile rural, Santiago. Chile. p. 247.

Render, B.; Stair, R. y Hanna, M.

2006 Métodos Cuantitativos para los negocios, $9^{\mathrm{a}}$ Ed. Pearson -Prentice Hall. México.

Siebert, H.

1969 Regional Economic Grow. Theory and policy. International Book Co.

Stohr, W.

1972 El desarrollo regional en América Latina. Experiencias y Perspectivas, Ediciones CIAP, Buenos Aires.

Sunkel, O.

1970 Desarrollo, subdesarrollo, dependencia y desequilibrios espaciales, Revista latinoamericana de Estudios Urbanos y Regionales, Vol. $1 \mathrm{~N}^{\mathrm{o}} 1$ EURE (CIDU), Universidad Católica. Chile.

Torres, A. y Acevedo, E.

2008 "El problema de la salinidad en los recursos suelo y agua que afectan el riego y cultivos en los valles de Lluta y Azapa", Idesia, Vol. 26, No 3, pp. 31-44.

Valderas, $\mathrm{R}$

1988 Análisis de sistemas Zonales. Facultad de Ciencias Agrarias y Forestales, Universidad de Chile. Chile. p. 272.

Valderas, R.

1997 Economía Regional y desarrollo Rural, Anales de la Universidad de Chile, Sexta Serie No 5, octubre de 1997.

Valderas, R.; Meyer, M. y Marchant, R.

2006 Estudio Preliminar de la relación Producto Interno Bruto y Exportaciones agrícolas, 2006, XI Congreso de Economistas Agrarios, Universidad de Talca, 16-17 de noviembre. 2006, Talca. Chile.

Valderas, R.; Meyer, M.; González, R.

2010 Cambio económico productivo en el sector agropecuario silvícola a nivel regional y nacional. XV Congreso de Economistas Agrarios, Antumapu, Universidad de Chile 14-15 de octubre 2010, Libro resúmenes presentación. p. 64.

Williamson, G.

1965 Regional inequality and the process of national development: A description of the pattern, Economic development and Cultural Change, vol. $13 \mathrm{~N}^{\circ} 4$. 